\title{
MULTIPLE TIMING OFFSETS COMPENSATION IN COOPERATIVE COMMUNICATION SYSTEMS
}

\author{
Xiao Li ${ }^{\dagger}$, Yik-Chung $W u^{\dagger}$ and Erchin Serpedin ${ }^{\star}$ \\ ${ }^{\dagger}$ Department of Electrical and Electronic Engineering, The University of Hong Kong, Hong Kong \\ * Department of Electrical and Computer Engineering, Texas A\&M University, College Station, USA \\ Email: \{lixiao,ycwu\}@eee.hku.hk, serpedin@ece.tamu.edu
}

\begin{abstract}
Cooperative communication systems have attracted much attention recently due to their desirable performance gain while using single antenna terminals. However, most of the existing works on cooperative communications require perfect timing synchronization among users, which is usually not true in practical systems. In this paper, a general framework of the re-synchronization filter design is developed in order to compensate the multiple timing offsets at the destination. The proposed method is applied to different scenarios with varying degrees of timing misalignment and is numerically shown to provide excellent performances that approach the perfectly synchronized case.
\end{abstract}

Index Terms - Cooperative, timing offset, resynchronization, Tikhonov regularization

\section{INTRODUCTION}

Nowadays, research involving distributed MIMO system, which suggests the sharing of antennas among single-antenna terminals to cooperatively transmit data, has received considerable attention because of its desirable cooperative diversity gain [1]-[2]. So far, the research over cooperative communication systems predominantly assumes perfect timing synchronization among users. However, many analytical and numerical results [7]-[8] have shown that timing errors in a cooperative system can jeopardize the reliability of communications. Especially, the benefit of cooperation would even vanish in terms of diversity gain [9] and system capacity [10] when the timing errors are large. All these results demonstrate that appropriate counter-measures to the asynchronous reception in distributed MIMO systems are absolutely necessary.

This paper develops a general framework for the multiple timing offsets compensation problem in cooperative systems. A re-synchronization filter is proposed to compensate the intersymbol interference (ISI) caused by the temporal asynchronism at the destination terminal. A general method to deal

This study was partially supported by a grant from the Research Grants Council of the Hong Kong SAR. with the re-synchronization filter design problem is developed by using the weighted regularized least squares (WRLS) approach, with the optimal regularization parameter chosen by the L-curve technique. It is shown that the symbol error rate (SER) after the compensation fundamentally improves and approaches the ideal case when the timing misalignment is relatively mild.

The rest of this paper is organized as follows. In Section 2, a general system model for the considered scenario is presented. Design of the re-synchronization filter is discussed in Section 3 with an in-depth analysis over the proposed method. Section 4 provides numerical results to validate the proposed re-synchronization scheme. Finally, the paper is concluded in Section 5.

Notation : The superscripts $(\cdot)^{*},(\cdot)^{H}$ and $(\cdot)^{T}$ denote the conjugate, the conjugate transpose and the transpose operators respectively. Notation $\mathbf{I}$ is the identity matrix, while $\|\mathbf{x}\|$ represents the $L_{2}$ norm of vector $\mathbf{x}$.

\section{SYSTEM MODEL}

In a cooperative system as shown in Fig. 1, the destination node (denoted as $\mathbb{D}$ ) receives signals from multiple distributed users (denoted as $\mathbb{R}_{k}$ ). Different $\mathbb{R}_{k}$ cooperatively transmit data to $\mathbb{D}$, where the common strategies include distributed space-time coding [7], and transmit maximal ratio combining (MRC) [9] etc., which requires perfect timing synchronization among users. However, due to the hardware inconsistencies and diverse locations, the signals from different $\mathbb{R}_{k}$ arriving at $\mathbb{D}$ are not synchronized to each other.

The received signal (within $0 \leq t \leq L_{o} T$ ) at $\mathbb{D}$ can be readily expressed as

$$
d(t)=\sum_{k=1}^{K} h_{k} \sum_{i=-L_{g}}^{L_{o}+L_{g}-1} r_{k}(i) g\left(t-i T-\epsilon_{k} T\right)+n(t)
$$

where $h_{k}$ is the complex channel coefficient from $\mathbb{R}_{k}$ to $\mathbb{D}$ and is assumed to be zero mean, circular complex Gaussian random variable with unit variance. The term $n(t)$ is the zero mean, circular complex additive white Gaussian noise 


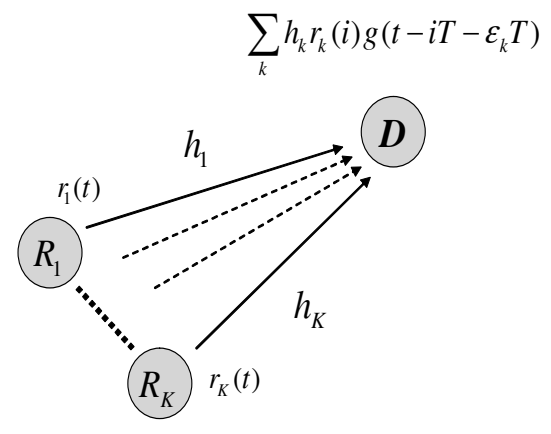

Fig. 1. A typical cooperative scenario

(AWGN) with variance $N_{o}$. Notation $T$ is the symbol duration, $r_{k}(i)$ is the complex valued symbol from $\mathbb{R}_{k}$ with symbol energy $\mathbb{E}\left\{\left|r_{k}(i)\right|^{2}\right\}=E_{k} ; \epsilon_{k} \in[0,1)$ is the unknown timing offset normalized to the symbol duration and $g(t)$ is the pulse shaping filter. Symbol $L_{o}$ represents the observation interval while $L_{g}$ is the approximated effective duration of the tail of $g(t)$ on one side.

Upon reception, the signal is oversampled at $\mathbb{D}$ by a ratio $Q \geq 2$ and thus the sample interval is $T_{s}=T / Q$. By stacking $L_{o} Q$ received samples, the received vector is given by [11]

$$
\mathbf{d}=\underbrace{\left[\mathbf{A}_{\epsilon_{1}} \mathbf{A}_{\epsilon_{2}} \cdots \mathbf{A}_{\epsilon_{K}}\right.}_{\triangleq \mathbf{A}_{\boldsymbol{\epsilon}}}] \underbrace{\left[\begin{array}{c}
h_{1} \mathbf{r}_{1} \\
h_{2} \mathbf{r}_{2} \\
\vdots \\
h_{K} \mathbf{r}_{K}
\end{array}\right]}_{\triangleq \boldsymbol{\Gamma}}+\mathbf{n}
$$$$
=\mathbf{A}_{\epsilon} \boldsymbol{\Gamma}+\mathbf{n}
$$

where

$$
\begin{aligned}
\mathbf{d} \triangleq & {\left[d(0), d\left(T_{s}\right), \cdots, d\left(\left(L_{o} Q-1\right) T_{s}\right)\right]^{T} } \\
\mathbf{n} \triangleq & {\left[n(0), n\left(T_{s}\right), \cdots, n\left(\left(L_{o} Q-1\right) T_{s}\right)\right]^{T} } \\
\mathbf{A}_{\epsilon_{k}} \triangleq & {\left[\mathbf{a}_{-L_{g}}\left(\epsilon_{k}\right), \cdots, \mathbf{a}_{0}\left(\epsilon_{k}\right), \cdots, \mathbf{a}_{L_{o}+L_{g}-1}\left(\epsilon_{k}\right)\right] } \\
\mathbf{a}_{i}\left(\epsilon_{k}\right) \triangleq & {\left[g\left(-i T-\epsilon_{k} T\right), g\left(-i T+T_{s}-\epsilon_{k} T\right),\right.} \\
& \left.\cdots, g\left(-i T+\left(L_{o} Q-1\right) T_{s}-\epsilon_{k} T\right)\right]^{T} \\
\mathbf{r}_{k} \triangleq & {\left[r_{k}\left(-L_{g}\right), \cdots, r_{k}(0), \cdots, r_{k}\left(L_{o}+L_{g}-1\right)\right]^{T} . }
\end{aligned}
$$

Note that the phase offsets between transmitters and receiver are not explicitly specified in the system model (1), because they can be incorporated into the unknown channel coefficients while keeping the formulation of the system model unchanged.

In this paper, it is assumed that $\epsilon_{k}$ and $h_{k}$ have been estimated and we focus on how to compensate the time asynchronism at $\mathbb{D}$.

\section{RESYNCHRONIZATION FILTER DESIGN}

At destination $\mathbb{D}$, asynchronous signals from different users $\mathbb{R}_{k}$ overlap with each other. Hence there is no unique optimal sampling instant since the optimal instants for distinct users are different.

To cope with this problem, one way is to treat the signals from asynchronous users as signals passing through different paths in a multipath channel, then a joint maximum likelihood sequence estimator (JMLSE) can be used to jointly decode the data from all the users [7]. Although JMLSE, in principle, can be applied to scenarios with any number of users, its implementation complexity becomes prohibitive when the number of users is greater than two.

A more direct method to resynchronize the multiple users is to design a re-synchronization filter at $\mathbb{D}$ to realign the asynchronous signals as first demonstrated in [12]. Unfortunately, general guidelines for choosing the optimal design parameters and comprehensive performance analysis studies are missing in [12]. In the following, we will present a general framework for the re-synchronization filter design and show that the scheme in [12] is a special case of the proposed framework.

\subsection{Problem Statement}

After the multiple timing offsets $\epsilon \triangleq\left[\epsilon_{1}, \cdots, \epsilon_{K}\right]^{T}$ and channels $\mathbf{h} \triangleq\left[h_{1}, \cdots, h_{K}\right]^{T}$ have been estimated at $\mathbb{D}$, the remaining issue is how to employ the estimates to compensate the offsets to obtain ISI-free reception. Mathematically, this problem can be solved by designing a re-synchronization filter $\mathbf{f}$ such that after the convolution with the received signal (1), the ISI components are minimized.

This process is actually an equalization for the fractional timing offset distortion and equivalent to the minimization problem

$$
\min _{\mathbf{f}} \mathbb{E}\left\{\left\|\hat{\boldsymbol{\Gamma}}^{H}\left(\mathbf{A}_{\hat{\boldsymbol{\epsilon}}}^{H} \mathbf{f}-\mathbf{b}\right)\right\|^{2}\right\},
$$

where the expectation is taken with respect to data realization. The vector $\mathbf{b} \triangleq\left[\boldsymbol{\delta}_{1}^{H}, \cdots, \boldsymbol{\delta}_{K}^{H}\right]^{H}$ and $\boldsymbol{\delta}_{1}=\cdots=\boldsymbol{\delta}_{K} \triangleq$ $\left[R_{g g}\left(-L_{g}-M_{o}\right), \cdots, R_{g g}(0), \cdots, R_{g g}\left(L_{g}+M_{o}\right)\right]^{H}$ stands for the ideal zero-ISI sampled waveform after matched filtering, where $M_{o}=\left(L_{o}-1\right) / 2$ (for the sake of discussion, $L_{o}$ is taken to be an odd number) and $R_{g g}(\tau)$ is the autocorrelation function of $g(t)$ at $t=\tau T$. The expression of $\hat{\boldsymbol{\Gamma}}$ in (2) is the $\Gamma$ in (1) with $h_{k}$ replaced by $\hat{h}_{k}$ and can be equivalently expressed as

$$
\hat{\boldsymbol{\Gamma}}=\underbrace{\left[\begin{array}{ccc}
\hat{h}_{1} \mathbf{I} & & \mathbf{0} \\
& \ddots & \\
\mathbf{0} & & \hat{h}_{K} \mathbf{I}
\end{array}\right]}_{\triangleq \mathbf{H}} \underbrace{\left[\begin{array}{c}
\mathbf{r}_{1} \\
\vdots \\
\mathbf{r}_{K}
\end{array}\right]}_{\triangleq \mathbf{r}}=\mathbf{H r} .
$$




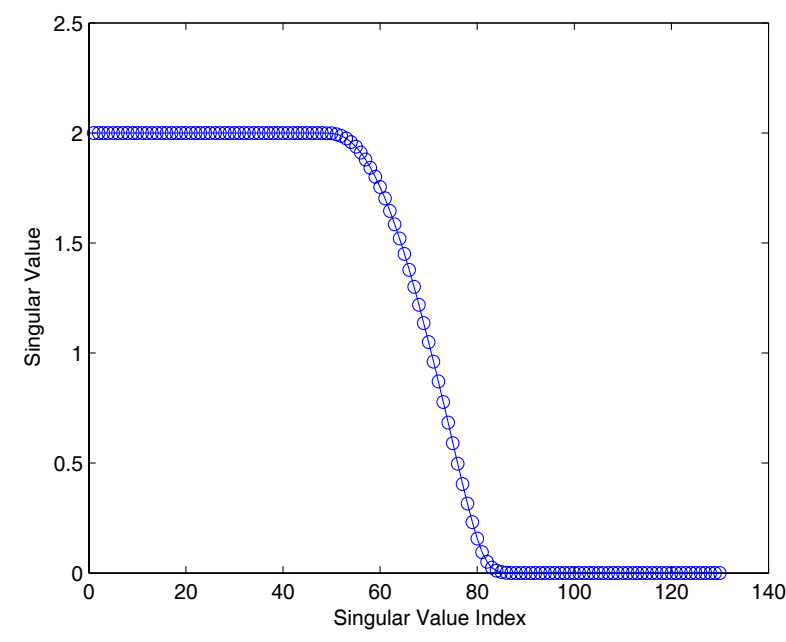

Fig. 2. An example of the singular values distribution of the ill-conditioned matrix $\mathbf{A}_{\boldsymbol{\epsilon}}$ with $L_{o}=65, L_{g}=4$ and $Q=2$

Thus, (2) can be reformulated as the following weighted least squares (WLS) problem

$$
\min _{\mathbf{f}}\left(\mathbf{A}_{\hat{\boldsymbol{\epsilon}}}^{H} \mathbf{f}-\mathbf{b}\right)^{H} \boldsymbol{\Pi}\left(\mathbf{A}_{\hat{\boldsymbol{\epsilon}}}^{H} \mathbf{f}-\mathbf{b}\right) .
$$

The symbol $\boldsymbol{\Pi} \triangleq \mathbf{H} \mathbf{R} \mathbf{H}^{H}$ represents the positive definite $m \times m$ weighting matrix with $m=K\left(L_{o}+2 L_{g}\right)$, and $\mathbf{R}=$ $\mathbb{E}\left\{\mathbf{r} \mathbf{r}^{H}\right\}$ is given by

$$
\mathbf{R} \triangleq\left[\begin{array}{ccc}
\mathbf{R}_{1,1} & \cdots & \mathbf{R}_{1, K} \\
\vdots & \ddots & \vdots \\
\mathbf{R}_{K, 1} & \cdots & \mathbf{R}_{K, K}
\end{array}\right]
$$

with the sub-matrix $\mathbf{R}_{i, j}=\mathbb{E}\left\{\mathbf{r}_{i} \mathbf{r}_{j}^{H}\right\}$ being the correlation matrix between $\mathbf{r}_{i}$ and $\mathbf{r}_{j}$. Note that once the transmission scheme and cooperative strategy are fixed, the correlation matrix can be determined and made available at both the transmitter and the receiver.

Generally speaking, the solution to (4) is the WLS solution $\mathbf{f}=\left(\mathbf{A}_{\hat{\boldsymbol{\epsilon}}} \boldsymbol{\Pi} \mathbf{A}_{\hat{\boldsymbol{\epsilon}}}^{H}\right)^{-1} \mathbf{A}_{\hat{\boldsymbol{\epsilon}}} \boldsymbol{\Pi} \mathbf{b}$. However, because the timing offsets can be represented as $\epsilon_{k}=\epsilon_{o}+\Delta_{k}$, where $\epsilon_{o}$ is the common offset and $\Delta_{k}$ includes the travel delay difference, the columns of $\mathbf{A}_{\hat{\epsilon}}^{H}$ are quite similar to each other. Hence, (4) is an ill-posed problem. As opposed to a rank-deficient problem, there are no general rules to determine which singular values of an ill-conditioned matrix to discard [14], as there is no significant gap between the singular values (an example of the singular value distribution of $\mathbf{A}_{\hat{\boldsymbol{\epsilon}}}^{H}$ is shown in Fig. 2), leading to the amplification of any perturbation that exists in the system.

In the following, we present a general framework that employs the regularization theory to design a filter which minimizes the residual ISI power.

\subsection{Regularized Filtering}

We hereby propose the weighted regularized least squares (WRLS) [15] (also known as scaled Tikhonov regularization) method to deal with the ill-posed problem in (4). To simplify our notation in the following analysis, the optimization problem in (4) is re-expressed compactly as

$$
\min _{\mathbf{f}}\left(\mathbf{G}_{\Pi} \mathbf{f}-\mathbf{b}_{\Pi}\right)^{H}\left(\mathbf{G}_{\Pi} \mathbf{f}-\mathbf{b}_{\Pi}\right)
$$

where $\mathbf{G}_{\Pi}=\boldsymbol{\Pi}^{\frac{1}{2}} \mathbf{A}_{\hat{\boldsymbol{\epsilon}}}^{H}$ and $\mathbf{b}_{\Pi}=\boldsymbol{\Pi}^{\frac{1}{2}} \mathbf{b}$, with $\boldsymbol{\Pi}^{\frac{1}{2}}$ representing the Cholesky square root of the weighting matrix $\Pi$. Now the WRLS problem can be stated as

$$
\min _{\mathbf{f}}\left[\left(\mathbf{G}_{\Pi} \mathbf{f}-\mathbf{b}_{\Pi}\right)^{H}\left(\mathbf{G}_{\Pi} \mathbf{f}-\mathbf{b}_{\Pi}\right)+\lambda^{2} \mathbf{f}^{H} \mathbf{L}^{H} \mathbf{L f}\right],
$$

where $\mathbf{f}^{H} \mathbf{L}^{H} \mathbf{L f}$ controls the properties of the regularized solution norm by choosing different regularization matrices $\mathbf{L}$ (e.g., identity matrix for minimum energy, first derivative for maximum flatness [14]); the variable $\lambda$ stands for the regularization parameter that balances the minimization of the two terms. When $\lambda=0$, formulation (6) reduces to the WLS problem in (4). In order to find the solution of (6), we rewrite (6) as

$$
\min _{\mathbf{f}}\left\|\left(\begin{array}{c}
\mathbf{G}_{\Pi} \\
\lambda \mathbf{L}
\end{array}\right) \mathbf{f}-\left(\begin{array}{c}
\mathbf{b}_{\Pi} \\
\mathbf{0}
\end{array}\right)\right\|^{2} .
$$

For a fixed $\lambda$, the solution is readily obtained as

$$
\mathbf{f}_{\lambda}=\left(\mathbf{G}_{\Pi}^{H} \mathbf{G}_{\Pi}+\lambda^{2} \mathbf{L}^{H} \mathbf{L}\right)^{-1} \mathbf{G}_{\Pi}^{H} \mathbf{b}_{\Pi} .
$$

Notice that in (7), if $\lambda$ is too large, the solution is overregularized and the residual error may be overwhelming, while if it is too small, the solution becomes under-regularized and the stability of the solution is highly affected. As can be seen, the performance of the regularized solution $\mathbf{f}_{\lambda}$ depends heavily on the regularization parameter $\lambda$ (the choice of $\mathbf{L}$ is relatively straightforward as different $\mathbf{L}$ present different physical meanings).

\subsubsection{Choice of Regularization Parameter $\lambda$}

As discussed previously, the value of $\lambda$ could significantly affect the performance of the re-synchronization filter. Therefore, choosing an appropriate $\lambda$ is very important. A conceptually simple tool for the analysis of discrete ill-posed problems is the L-curve technique [14], which is a plot of the discrete smoothing norm $\left\|\mathbf{L f}_{\lambda}\right\|^{2}$ of the regularized solution versus the corresponding residual norm $\left\|\mathbf{G}_{\Pi} \mathbf{f}_{\lambda}-\mathbf{b}_{\Pi}\right\|^{2}$ for different $\lambda$. The L-curve clearly displays the compromise between minimization of these two quantities as illustrated in Fig. 3 (with $\mathbf{L}=\mathbf{I}$ ).

The L-curve corner, defined as the maximum curvature point on the curve $\left(\log \left\|\mathbf{G}_{\Pi} \mathbf{f}_{\lambda}-\mathbf{b}_{\Pi}\right\|, \log \left\|\mathbf{L f}_{\lambda}\right\|\right)$, appears to be a good compromise that balances the regularization errors and perturbation errors in the regularized solution 


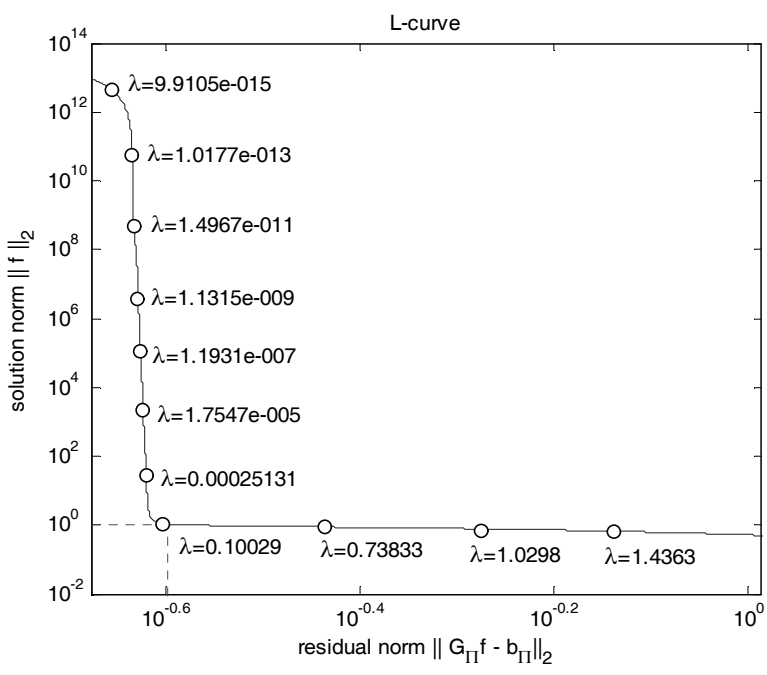

Fig. 3. An example of L-curve

$\mathbf{f}_{\lambda}$ [14]. Therefore, we will take this point for the regularization parameter $\lambda$. In order to find the $\lambda$ that corresponds to the L-curve corner, let $\rho=\left\|\mathbf{G}_{\Pi} \mathbf{f}_{\lambda}-\mathbf{b}_{\Pi}\right\|$ and $\xi=\left\|\mathbf{L f}_{\lambda}\right\|$. Then the curvature $\kappa(\lambda)$ is given by [14]

$$
\kappa(\lambda)=2 \frac{\xi \rho}{\xi^{\prime}} \frac{\lambda^{2} \xi^{\prime} \rho+2 \lambda \xi \rho+\lambda^{4} \xi \xi^{\prime}}{\left(\lambda^{2} \xi^{2}+\rho^{2}\right)^{3 / 2}}
$$

where $\xi^{\prime}=4 \mathbf{f}_{\lambda}^{H}\left(\mathbf{G}_{\Pi}^{H} \mathbf{G}_{\Pi}+\lambda^{2} \mathbf{L}^{H} \mathbf{L}\right)^{-1} \mathbf{G}_{\Pi}^{H}\left(\mathbf{G}_{\Pi} \mathbf{f}_{\lambda}-\mathbf{b}_{\Pi}\right) / \lambda$. Note that when channel and timing offset realizations are given, $\rho, \xi$ and $\xi^{\prime}$ can be computed. Therefore, the regularization parameter $\lambda$ with maximum curvature in (8) can be located (e.g., by exhaustive search).

\subsubsection{Special Case - Least Squares with a Quadratic Con- straint}

The re-synchronization filter design was first discussed in [12] using the LS with a quadratic constraint (the Fixed SNR Loss or the Fixed Fidelity Loss methods). Note that there is no weighting matrix $\Pi$ in the original method in [12]. In the following, we incorporate the weighting matrix $\Pi$ to make the discussion more general, and setting $\Pi=\mathbf{I}$ will reduce the formulation back to that of [12]. The methods in [12] can be stated as

- Fixed SNR Loss Requirement

$$
\min \left\|\mathbf{G}_{\Pi} \mathbf{f}-\mathbf{b}_{\Pi}\right\|^{2} \quad \text { s.t. } \quad\|\mathbf{L f}\|^{2} \leq \alpha
$$

- Fixed Fidelity Loss Requirement

$$
\min \|\mathbf{L f}\|^{2} \quad \text { s.t. } \quad\left\|\mathbf{G}_{\Pi} \mathbf{f}-\mathbf{b}_{\Pi}\right\|^{2} \leq \beta .
$$

The constraints $\alpha$ and $\beta$ are design parameters that bound the norm of the solution and the errors respectively. In the following, we derive the relationship between the regularization framework and these two methods. In order to do this, the generalized singular value decomposition (GSVD) of the matrix pair $\left(\mathbf{G}_{\Pi}, \mathbf{L}\right)$ is needed

$$
\mathbf{G}_{\Pi}=\mathbf{U} \mathbf{\Sigma} \mathbf{Y}^{-1}, \quad \mathbf{L}=\mathbf{V M Y}^{-1}
$$

where $\boldsymbol{\Sigma}=\operatorname{diag}\left(\sigma_{1}, \cdots, \sigma_{n}\right), \mathbf{M}=\operatorname{diag}\left(\mu_{1}, \cdots, \mu_{n}\right)$ are the singular values of $\mathbf{G}_{\Pi}$ and $\mathbf{L}$ respectively with $n=L_{o} Q$. Matrices $\mathbf{U}$ and $\mathbf{V}$ represent the unitary singular vector matrices of $\mathbf{G}_{\Pi}$ and $\mathbf{L}$ (i.e. $\mathbf{U}^{H} \mathbf{U}=\mathbf{V}^{H} \mathbf{V}=\mathbf{I}$ ), while $\mathbf{Y}$ is a nonsingular matrix that satisfies $\mathbf{Y}^{H} \mathbf{G}_{\Pi}^{H} \mathbf{G}_{\Pi} \mathbf{Y}=\boldsymbol{\Sigma}^{2}$ and $\mathbf{Y}^{H} \mathbf{L}^{H} \mathbf{L Y}=\mathbf{M}^{2}$. With the GSVD of $\left(\mathbf{G}_{\Pi}, \mathbf{L}\right)$, the regularized solution $\mathbf{f}_{\lambda}(7)$ is expressed as

$$
\mathbf{f}_{\lambda}=\mathbf{Y}\left(\boldsymbol{\Sigma}^{2}+\lambda^{2} \mathbf{M}^{2}\right)^{-1} \boldsymbol{\Sigma} \mathbf{U}^{H} \mathbf{b}_{\Pi} .
$$

For the WLS with Fixed SNR Loss requirement (9), the solution would occur on the boundary $\|\mathbf{L f}\|^{2}=\alpha$ [12]. Substituting (12) into the boundary, we have

$$
\left\|\mathbf{V M} \mathbf{Y}^{-1} \cdot \mathbf{Y}\left(\boldsymbol{\Sigma}^{2}+\lambda^{2} \mathbf{M}^{2}\right)^{-1} \boldsymbol{\Sigma} \mathbf{U}^{H} \mathbf{b}_{\Pi}\right\|=\alpha .
$$

After some tedious but straightforward manipulations, the relationship between $\lambda$ and $\alpha$ is given by

$$
\sum_{i=1}^{n} \frac{\left(\sigma_{i} \mu_{i}\right)^{2}}{\left(\sigma_{i}^{2}+\lambda^{2} \mu_{i}^{2}\right)^{2}} \mathbf{b}_{\Pi}^{\star}(i)^{2}=\alpha^{2}
$$

where $\mathbf{b}_{\Pi}^{\star}=\mathbf{U}^{H} \mathbf{b}_{\Pi}$. From (13), it can be seen that once a threshold value $\alpha$ is specified, there exists a corresponding $\lambda$ in the regularization framework.

For the WLS with Fixed Fidelity Loss requirement (10), we can follow the same method to obtain a similar relationship as

$$
\sum_{i=1}^{n}\left(\frac{\mu_{i}^{2} \mathbf{b}_{\Pi}^{\star}(i)}{\tilde{\lambda}^{2} \sigma_{i}^{2}+\mu_{i}^{2}}\right)^{2}=\beta^{2}, \quad \text { with } \quad \tilde{\lambda}=\frac{1}{\lambda}
$$

From (13) and (14), it is clear that WLS with a quadratic constraint method is a special case of the proposed framework when the constraint values $\alpha, \beta$ are optimally chosen.

Although WLS with a quadratic constraint methods are equivalent to the regularization method under some circumstances, generally there is no guideline for choosing optimal $\alpha$ and $\beta$. Furthermore, because the elements of $\mathbf{G}_{\Pi}$ depend on different timing offset and channel realizations, there is no single $\lambda$ that is in general optimized for all the scenarios. Thus, fixing the values of $\alpha$ and $\beta$ in LS with a quadratic constraint as in [12] can fail substantially (more details can be found in the next section). 


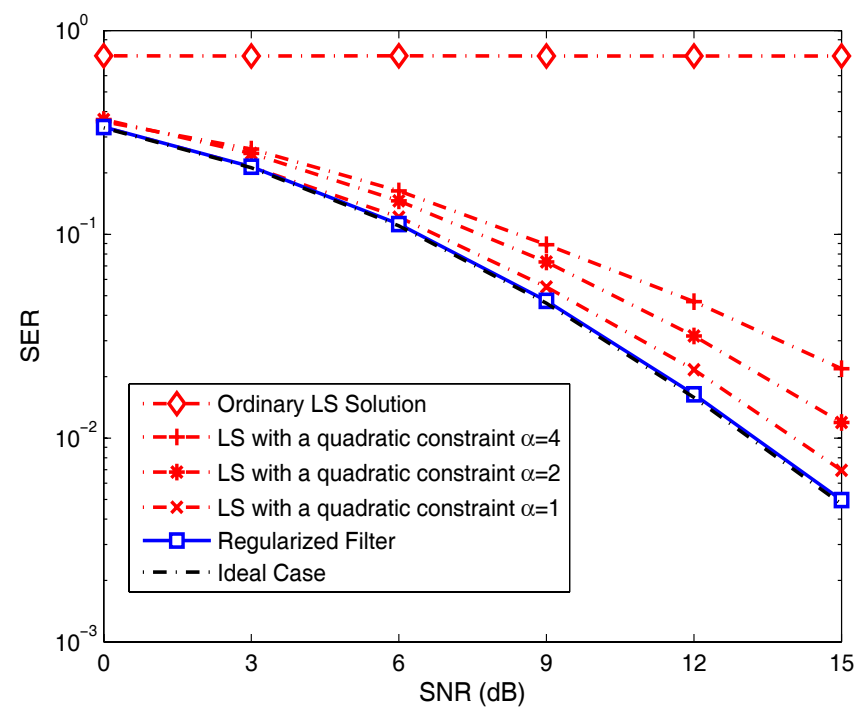

Fig. 4. Symbol Error Rate (SER) performance for resynchronization filter designed by Weighted Regularized LS against the performance by ordinary LS solution, and LS with a quadratic constraint with $\alpha=4,2,1$ under QPSK modulation, $\left|\Delta_{k}\right|<0.1 T$ for $K=2$.

\section{NUMERICAL RESULTS AND DISCUSSIONS}

In this section, the performance of the proposed resynchronization filter is demonstrated by Monte Carlo simulations, where each point is obtained by averaging over $10^{4}$ runs. In all simulations, the QPSK modulation is used. Due to the space limit, only the results under the scenario using spacetime block coding (STBC) are shown in this paper because other schemes such as transmit maximal ratio combining (MRC) provide similar results.

Specifically, Alamouti Scheme is employed for the $K=$ 2 case, and the rate- $1 / 2$ orthogonal STBC for four antennas [13] is used for the $K=4$ case to cooperatively transmit data. Thus the symbol energy of each $\mathbb{R}_{k}$ should satisfy $E_{1}=$ $\cdots=E_{K} \triangleq E_{R}$. In both cases, it can be easily shown that the correlation matrix is diagonal as $\mathbf{R}=E_{R} \mathbf{I}$ and the weighting matrix becomes

$$
\boldsymbol{\Pi} \triangleq E_{R}\left[\begin{array}{ccc}
\left|\hat{h}_{1}\right|^{2} \mathbf{I} & & \mathbf{0} \\
& \ddots & \\
\mathbf{0} & & \left|\hat{h}_{K}\right|^{2} \mathbf{I}
\end{array}\right] .
$$

The pulse shaping filter $g(t)$ is assumed as root-raised cosine waveform with roll-off factor 0.22 and normalized en$\operatorname{ergy} \int_{-\infty}^{\infty} g^{2}(t) d t=1$. The regularization matrix $\mathbf{L}$ is taken to be $\mathbf{I}$ such that $\|\mathbf{L f}\|^{2}$ represents the energy of the filter. The channel coefficients are modeled as independent identically distributed (i.i.d.) complex Gaussian random variables

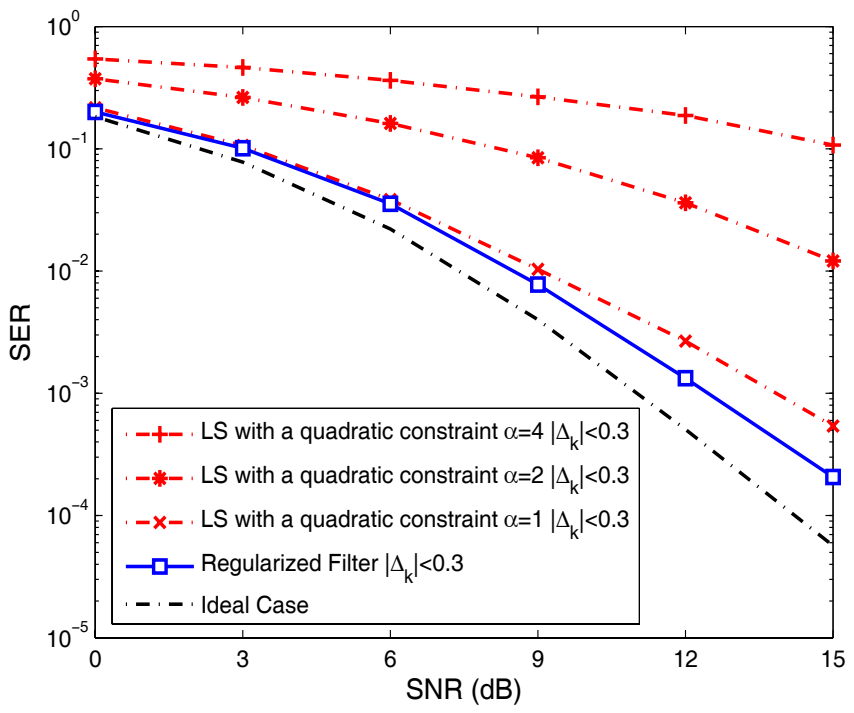

Fig. 5. Symbol Error Rate (SER) performance of the Regularized Filter and the filter designed by LS with a quadratic constraint with $\alpha=4,2,1$ under QPSK modulation, $\left|\Delta_{k}\right|<$ $0.3 T$ for $K=4$.

with zero mean and unit variance. The SNR is defined as the average transmit signal-to-noise ratio as $\mathrm{SNR}_{D}=E_{R} / N_{o}$ at $\mathbb{D}$. The block length is fixed to $L_{o}=65$ and $L_{g}=4$.

In Fig. 4, the performance of the proposed regularized filter is illustrated against several LS methods with $K=2$ and travel delay differences $\left|\Delta_{k}\right|<0.1 T$, where the $\Delta_{k}$ is generated uniformly between -0.1 to 0.1 for each simulation run. It can be seen that the performance of the regularized filter overlaps with the ideal case (i.e., $\Delta_{k}=0$ ) while the ordinary LS solution provides disappointing results due to the illconditioned nature of the problem. On the other hand, for the LS with a quadratic constraint [12], it is obvious that the nonoptimal quadratic constraint parameters $\alpha$ lead to significant performance degradation, especially in the case with $\alpha=4$. Because similar results are obtained for both the Fixed SNR Loss method and the Fixed Fidelity Loss method, only the performances of Fixed SNR Loss method are presented.

In Fig. 5, the regularized filter is compared against the LS methods with $K=4$ and travel delay differences up to $\left|\Delta_{k}\right|<0.3 T$. When the timing mismatch is severe, the choice of $\alpha$ becomes more critical for the LS filter. For example, it is observed that the performance gaps between the LS filters with different $\alpha$ are much larger than those in the previous case with a small $\Delta_{k}$. On the other hand, the proposed regularized filter with optimally located parameter $\lambda$ still remains close to the ideal case, outperforming the LS filters with parameters $\alpha$ chosen empirically or in an ad hoc way. 


\section{CONCLUSIONS}

In this paper, the re-synchronization of multiple timing offsets in a cooperative system has been considered. We have unified the framework of re-synchronization filter design and derived the relationship between LS filter with a quadratic constraint and the regularized filter. Compared to the existing method, the proposed framework automatically determines the optimal design constraints given different realizations of channels and offsets. Numerical results have illustrated that the proposed filter outperforms the existing method no matter under mild or severe timing mismatch, which salvages the performance loss due to temporal asynchronism.

\section{REFERENCES}

[1] A. Sendonaris, E. Erkip and B. Aazhang, "User Cooperation Diversity, Part I, II", IEEE Trans. on Commun., vol. 51, pp. 1927-1948, Nov. 2003.

[2] J.N. Laneman and G.W. Wornell, "Distributed SpaceTime-Coded Protocols for Exploiting Cooperative Diversity in Wireless Networks", IEEE Trans. Inform. Theory, vol. 49, no. 10, pp. 2415-2425, Oct 2003

[3] P.A. Anghel and M. Kaveh, "Exact Symbol Error Probability of a Cooperative Network in a Rayleigh-Fading Environment", IEEE Trans. Wireless Commun., vol. 3, pp. 1416-1421, Sept. 2004.

[4] A. Ribeiro, X. Cai, G.B. Giannakis, "Symbol Error Probabilities for General Cooperative Links", IEEE Trans. Wireless Commun., vol. 4, pp. 1264-1273, May. 2005 .

[5] R.U. Nabar and H. Bolcskei, "Space-Time Signal Design for Fading Relay Channels", Proc. GlobeCom, pp. 1952-1956, Dec. 2003.

[6] O.-S. Shin, A.M. Chan, H.T. Kung, V. Tarokh, "Design of an OFDM Cooperative Space-Time Diversity System", IEEE Trans. on Commun., vol. 56, no. 4, pp. 2203 - 2215, Jul. 2007.

[7] J. Mietzner and P.A. Hoeher, "Distributed Space-Time Codes for Cooperative Wireless Networks in the Presence of Different Propagation Delays and Path Losses", Proc. Third IEEE Sensor Array and Multichannel Signal Processing Workshop, pp. 264-268, Barcelona, Spain, Jul. 2004.

[8] R.C. Palat, A. Annamalai and J.H. Reed, "Upper Bound on Bit Error Rate for Time Synchronization Errors in Bandlimited Distributed MIMO Networks", Proc. IEEE WCNC, vol. 4, pp. 2058-2063, Apr. 2006.
[9] S. Jagannathan, H. Aghajan, A. Goldsmith, "The Effect of Time Synchronization Errors on the Performance of Cooperative MISO Systems", Proc. IEEE Globecom, pp. 102-107, Nov. 2004.

[10] R.A. Iltis and R. Cagley, "Channel Estimation and Carrier Offset Control for Cooperative MIMO Sensor Networks", IEEE Proc. 39th Asilomar Conf. Signals, Systems, Computing, vol. 1, Pacific Grove, CA, pp. 210214, Nov. 2005.

[11] Y.-C. Wu and E. Serpedin, "Design and Analysis of Feedforward Symbol Timing Estimators Based on the Conditional Maximum Likelihood Principle", IEEE Trans. Signal Processing, vol. 53, no. 5, pp. 1908-1918, May. 2005.

[12] A. Kannan, T.P. Krauss and M.D. Zoltowski, "Separation of Co-channel Signals Under Imperfect Timing and Carrier Synchronization", IEEE Trans. Veh. Tech., vol. 50, pp. 79-96, Jan. 2004.

[13] E.G. Larsson and P. Stoica, Space-Time Block Coding for Wireless Communications, Cambridge Unviersity Press,2003.

[14] P.C. Hansen, Rank Deficient and Discrete Ill-Posed Problems-Numerical Aspects of Linear Inversion, SIAM, 1998.

[15] Ali H. Sayed, Fundamentals of Adaptive Filtering, Wiley-IEEE Press, 2003. 